\title{
Healthy Immigrant Effect: Trayectoria de salud de la población inmigrante a partir de la ENSE 2011-2012
}

\author{
Berta Rivera a , BRUNO CASAL ${ }^{\text {a,b }}$, LUIS CURRAIS ${ }^{\text {a }}$ \\ a Universidad de A Coruña, Facultad de Economía y Empresa, Campus de Elviña, s/n, 15071 A \\ Coruña,España.E-mail: berta@ucd.es, currais@udc.es \\ ${ }^{b}$ Universidad de A Coruña, E.U. de Relaciones Laborales, c/ Leopoldo Alas “Clarín”, 2, 15008 A \\ Coruña, España.E-mail: bruno.casal@udc.es
}

\begin{abstract}
RESUMEN
El objetivo principal de este artículo es estudiar la dinámica de salud de la población inmigrante en España y aportar evidencia empírica que demuestre la existencia del Healthy Immigrant Effect. Se utilizan datos de la Encuesta Nacional de Salud de España 2011-2012 y se estiman diferentes modelos probabilísticos, tomando como variables de resultado indicadores de salud autopercibida, presencia de trastornos crónicos y limitaciones de actividad. Los resultados obtenidos indican que la población inmigrante presenta un mejor estado de salud que la nacional. Se obtiene también evidencia a favor del Healthy Immigrant Effect, dado que el estado de salud tiende a empeorar con el tiempo de residencia en el país, convergiendo hacia los niveles de salud de la población nacional. Desde el punto de vista de los sistemas sanitarios, el estudio de las diferencias en salud en la población extranjera, y su evolución temporal, se muestra relevante para garantizar el acceso de la población inmigrante a los diferentes servicios de salud y atender a unas necesidades que varían con el tiempo transcurrido desde que se produce la migración.
\end{abstract}

Palabras clave: Healthy Immigrant Effect; estado de salud, inmigrante, años de inmigración.

\section{Healthy Immigrant Effect: An Empirical Approach to the Health Trajectory of Immigrant Population Based on the 2011-2012 ENSE}

\begin{abstract}
The main aim of this article is to study the health dynamics of the immigrant population in Spain and provide empirical evidence which proves the existence of the Healthy Immigrant Effect. Data from the 2011-2012 Spanish National Health Survey is used to estimate different probabilistic models, taking as outcome variables the self-assessed health status, the presence of chronic conditions and limitations in main activity. Our results show that the immigrant population has a better level of health than their Spanish counterparts. Evidence in favour of the Healthy Immigrant Effect is also found, since the health status tends to decline with length of time since immigration and to converge to native-born levels. From the point of view of health systems, the analysis of immigrant health differences and its trends is essential to meet the healthcare needs. It must be pointed that these necessities tend to vary with time since migration. Consequently, immigrants' access to different health services should be guaranteed.
\end{abstract}

Keywords: Healthy Immigrant Effect; Health Status, Immigrant, Years Since Immigration.

Clasificación JEL: I12, J10, J61

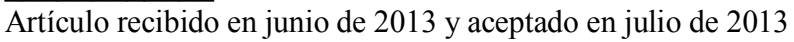

Artículo disponible en versión electrónica en la página www.revista-eea.net, ref. ə-31216

ISSN 1697-5731 (online) - ISSN 1133-3197 (print) 


\section{INTRODUCCIÓN}

La situación de crisis económica ha provocado que el colectivo de inmigrantes haya visto reducido su peso sobre el total de población española en, aproximadamente, medio punto porcentual anual desde el año 2010. Sin embargo, de acuerdo con la última explotación estadística del padrón municipal, la población extranjera continúa representando una parte importante de la misma, situándose en el $11,73 \%$ del total de población empadronada, lo que supone, en enero de 2013, unos 5,5 millones de personas (INE, varios años).

Si tenemos en cuenta que en 1996 esta población sólo alcanzaba el medio millón de individuos ( $1,37 \%$ del total de la población), podemos afirmar que la inmigración es un fenómeno relativamente reciente en España (INE, varios años). Desde finales de la década de los noventa, España se convierte en un país receptor de importantes flujos migratorios, procedentes de países con unas condiciones de salud muy diferentes a las nuestras (Solsona y Viciana, 2004). En general, podemos hablar de un perfil de inmigración de tipo económico, caracterizada por tratarse de una población joven, concentrada en cohortes en edad de trabajar (el 48\% de los extranjeros se sitúan en el tramo de edad de 25-45 años) y diversificada en cuanto a su origen (26\% procedentes de Latinoamérica, $17 \%$ de África y un $46 \%$ de la UE).

La intensificación y diversificación de los flujos migratorios plantea importantes retos para los sistemas de salud, al tener que mejorar el conocimiento sobre las características particulares de los inmigrantes y garantizar el acceso a los servicios de atención primaria y especializada (Cantarero y Pascual, 2008). En este contexto, se hace necesario abordar el estudio de la dinámica del estado de salud de esta población, con el fin de dar respuesta a sus necesidades y establecer prioridades en materia de atención sanitaria.

El tiempo residiendo en el país de destino, ha sido abordado por la literatura como uno de los factores condicionantes del estado de salud de la población inmigrante. El efecto conocido como Healthy Immigrant Effect (HIE), explicaría la dinámica de salud de la población extranjera desde su llegada a España. El HIE indica que los inmigrantes recién llegados al país de destino presentan, en comparación con la población nacional de similares características sociodemográficas, un mejor estado de salud, y este acabará convergiendo al nivel de la población autóctona a medida que aumentan los años de residencia.

El HIE es un fenómeno ampliamente estudiado en países que presentan una larga trayectoria en ser receptores de inmigrantes, como puede ser el caso de Estados Unidos, Canadá o Australia, sin embargo, en países en los que el fenómeno de la inmigración es reciente, como es el caso de España, el estudio de la existencia del $H I E$ es un aspecto escasamente abordado en la literatura. Ello está motivado, principalmente, por la ausencia de datos de tipo longitudinal sobre este colectivo, o información específica sobre periodos de residencia desde que 
se produce la migración. La Encuesta Nacional de Salud de España (ENSE), principal fuente de información utilizada en estudios que tienen por objeto el análisis del estado de salud de la población inmigrante, y el acceso y utilización de los servicios sanitarios, no recogía información sobre los años de residencia de la población extranjera en el país, en oleadas previas a la del año 2011. Sin embargo, en la ENSE 2011-2012 el cuestionario de adultos recoge esta información, permitiendo realizar una aproximación al comportamiento de diferentes indicadores de salud en función del número de años transcurridos desde que se produce la migración.

El objetivo principal de este artículo es analizar los determinantes del estado de salud de la población inmigrante en España y aportar evidencia empírica sobre la existencia del HIE, que explicaría la dinámica de salud de la población extranjera desde su llegada a nuestro país. Para el análisis empírico se utilizarán datos de la ENSE 2011-2012 y se tomarán, como variables del estado de salud, los indicadores de salud autopercibida, el padecimiento de enfermedades crónicas y la declaración de limitaciones para la realización de actividades de la vida diaria. El análisis se desarrollará considerando que los perfiles y la dinámica de salud de los inmigrantes estarán determinados por el tiempo de residencia en el país de destino, además de otras variables de control que tendrán en cuenta características socioeconómicas del individuo.

El artículo se estructura en cinco secciones. Después de revisar la bibliografía más relevante que aborda el estudio de la dinámica de salud de la población inmigrante, se presenta la metodología econométrica para el análisis empírico que se plantea posteriormente. Asimismo, se describe la ENSE y las variables de interés para las estimaciones. En la siguiente sección se presentan los principales resultados de las estimaciones econométricas realizadas para explicar la trayectoria de salud de la población extranjera en España. El artículo finaliza con una exposición de las principales conclusiones obtenidas en el estudio.

\section{EVIDENCIA PREVIA}

El proceso migratorio supone moverse de un entorno que presenta unos determinados parámetros de salud, en cuanto a comportamientos de riesgo, hábitos de vida, cultura sanitaria o acceso a servicios sanitarios, hacia otro con una combinación diferente de los mismos. La literatura que aborda el estudio de las diferencias que en términos de salud presenta la población extranjera respecto a la del país de acogida, y los determinantes de estas diferencias, es diversa y no del todo concluyente. La mayoría de las investigaciones se centran en el estudio de la trayectoria de salud de la población extranjera en países que presentan una larga experiencia en recibir flujos de inmigrantes, y en ella se confirma que la dinámica de salud del inmigrante se caracteriza por una ventaja inicial en términos de salud (Health Selectivity, HS), y una convergencia hacia los niveles de 
salud de la población nacional a medida que transcurre el tiempo de residencia en el país de acogida (HIE) (House et al., 1990; Stephen et al., 1994). Este empeoramiento en el estado de salud se advierte con una mayor intensidad en aquella inmigración que procede de regiones que presentan, respecto al país de destino, peores indicadores de mortalidad y morbilidad (Kennedy et al., 2006).

En la literatura no existe una única teoría a la existencia del HIE, sino diferentes explicaciones que pueden considerarse como complementarias entre sí (Frisbie et al., 2001). Posibles explicaciones a la trayectoria de salud del inmigrante son, por una parte, la existencia de una selección positiva en términos de salud (HS) (Marmot et al., 1984; McDonald y Kennedy, 2004; Jasso et al., 2004). De este modo, los individuos que presentan un mejor estado de salud son los más capacitados para emigrar, tanto física como financieramente. Al mismo tiempo, los inmigrantes que presentan peores estados de salud tienen una mayor probabilidad de retornar a su país de origen (Palloni y Arias, 2003; Palloni y Morenoff, 2001). Así por ejemplo, Marmot et al., (1984) demuestran que los inmigrantes asentados en Reino Unido presentan tasas estandarizadas de mortalidad inferiores a sus países de procedencia.

Autores como Jasso et al., (2004) y McDonald y Kennedy (2004) explican esta dinámica de salud en base a la percepción que tiene esta población sobre sus problemas de salud, y a la accesibilidad a los servicios sanitarios en el país de destino. Los inmigrantes pueden no declarar sus condiciones de salud a la llegada al país, y a medida que transcurre el tiempo de residencia declarar peores estados de salud porque aumenta el número de diagnósticos sobre condiciones de salud preexistentes. Por otro lado, también se puede entender que un mejor acceso a servicios sanitarios también puede repercutir en un mejor estado de salud, debido a un mayor uso de servicios de medicina preventiva, diagnósticos y tratamientos a sus problemas de salud (Leclere et al., 1994; Laroche, 2000; McDonald y Kennedy, 2004).

La exposición a factores ambientales comunes, y la existencia de un proceso de asimilación cultural repercuten en una identificación con comportamientos y estilos de vida similares a los de la población nacional, como puede ser la dieta o el ejercicio físico (Marmot y Syme, 1976; Stephen et al., 1994; Hyman, 2001). Diferentes estudios muestran la tendencia a que los inmigrantes procedentes de sociedades menos avanzadas tengan estilos de vida más saludables que la población del país de acogida, presentando por ello también mayores factores de riesgo ante hábitos perjudiciales como el consumo de tabaco y alcohol, o en la adopción de dietas menos saludables (Wister, 2005; Frisbie et al., 2001; Hamilton y Hummer, 2011). Un indicador utilizado en la literatura para capturar este efecto es el del Índice de Masa Corporal (IMC). En países como Estados Unidos se observa que los niveles de obesidad son inferiores a los de la 
población nacional en inmigrantes recién llegados al país, y que estos aumentan según el tiempo de residencia (Goel et al., 2004).

También debemos considerar la influencia que tienen sobre el estado de salud determinadas condiciones socioeconómicas, como son el nivel de educación y la situación laboral (Gómez et al., 2004; Marmot y Syme, 1976), y también condiciones de tipo genético (Marmot et al., 1984). En cuanto a la asimilación en términos de renta con la población nacional, es preciso puntualizar que si la salud está relacionada de forma positiva con el nivel de renta (Sorlie et al., 1995) y la mayoría de inmigrantes que llegan al país de destino presentan niveles de renta y ratios de empleo inferiores a la población nacional, con el tiempo de residencia en el país los niveles de salud tenderían a mejorar (efecto contrario al $H I E)$.

Existe un amplio cuerpo de literatura que estudia el HIE y que obtienen resultados consistentes para países con una larga trayectoria en ser receptores de población inmigrante, como es el caso de Estados Unidos, Canadá, Reino Unido o Australia. Entre otros, autores como Biddle et al., (2007), Chen et al., (1996), Newbold y Danforth (2003), Pérez (2002), Stephen et al., (1994), McDonald y Kennedy (2004), o Antecol y Bedard (2005), obtienen evidencia suficiente sobre la existencia del HIE. McDonald y Kennedy (2004) y Pérez (2002) obtienen que los inmigrantes a su llegada a Canadá presentan una menor incidencia de enfermedades crónicas, pero esta incidencia se aproxima a los niveles observados para la población nacional a medida que transcurre el tiempo de estancia en el país. Resultado similar obtiene Jasso et al., (2004) para la inmigración en los Estados Unidos. A pesar de haber algún estudio que no encuentra evidencia suficiente sobre el HIE, como puede ser el trabajo de Laroche (2000) o el de Dunn y Dyck (2000), su existencia está ampliamente aceptada en la literatura (Biddle et al., 2007).

Son escasos los estudios que analicen el HIE en países en los que el fenómeno de la inmigración sea relativamente reciente, como puede ser el caso de España. La mayoría de estos estudios únicamente comparan los estados de salud de la población inmigrante con una muestra de población nacional de similares características demográficas.

En un trabajo reciente, Nolan (2012) investiga la existencia del HIE para Irlanda, utilizando datos de la Survey of Lifestyle, Attitudes and Nutrition (SLAN) del año 2007. Los resultados obtenidos presentan evidencia muy limitada sobre la existencia del HIE para el caso de los indicadores de estado de salud autopercibido y la presencia de limitaciones en las actividades de la vida diaria. En el caso del indicador de salud que recoge el padecimiento de problemas de salud crónicos, los resultados no muestran la existencia del HIE. En estos resultados, es importante destacar la naturaleza de la inmigración que recibe Irlanda, por 
tratarse de una población procedente principalmente del Reino Unido o de países europeos culturalmente muy similares a Irlanda.

En el caso de España, Carrasco et al., (2007), a partir de la ENSE 2003, estudian los perfiles de salud, estilos de vida y la utilización de recursos sanitarios por la población inmigrante residente en España. Los autores no encuentran diferencias significativas en la distribución de la comorbilidad o en el estado de salud autopercibido entre la población inmigrante y la nacional. En cuanto a los hábitos de vida (consumo de alcohol y tabaco), la población inmigrante presenta mejores parámetros de estilos de vida que la nacional.

García-Gómez (2007) utiliza la encuesta de salud de Cataluña de 2006 y determina que, si bien el estado físico de los inmigrantes es mejor que el de la población nacional, la relación se invierte en el caso de la salud mental. La autora encuentra evidencia a favor del HIE para el indicador de estado de salud mental (General Household Questionnnaire, GHQ) y para la escala visual analógica de valoración del estado de salud.

Rivera et al., (2008), a partir de datos de la ENSE 2003, la Encuesta Europea de Renta y Condiciones de Vida (ECV) 2004 y 2005, y el Panel de Hogares de la Unión Europea (PHOGUE), aproximan los perfiles comparados de salud entre las poblaciones nacional y extranjera, así como el acceso a recursos sanitarios. Los resultados no permiten confirmar de manera consistente que la población inmigrante establecida en España posea un perfil epidemiológico muy diferenciado de la población nacional, si bien el indicador de salud autopercibida (ECV y ENSE), e indicadores objetivos de padecimiento de enfermedades crónicas (ENSE), muestran evidencias sobre un mejor estado de salud comparado para la población extranjera.

Hernández-Quevedo y Jiménez-Rubio (2009) utilizan las ENSE 2003 y 2006 para determinar si existen desigualdades en salud y en el acceso a servicios sanitarios entre la población nacional y la población extranjera. Como principales resultados obtienen que el nivel de salud declarado por el inmigrante dependerá de su región de origen, presentando un mejor estado de salud que la población nacional los inmigrantes de la $U E$, y un peor estado de salud los procedentes de Latinoamérica. Para los restantes orígenes no aprecian diferencias importantes.

\section{METODOLOGÍA Y POBLACIÓN OBJETO DE ESTUDIO}

\section{Diseño}

El presente artículo toma como marco teórico de referencia el trabajo de Jasso et al., (2004), en el que se presenta un modelo básico que explica la selección positiva del inmigrante en términos de salud y los mecanismos de su posterior trayectoria de salud en el país de destino. 
El análisis econométrico se realiza tomando, como variables del estado de salud del inmigrante, el indicador de salud autopercibida, la declaración de problemas de salud crónicos y la de limitaciones para la realización de actividades de la vida diaria. Sobre estas variables de resultado se aplican diferentes modelos de estimación probabilísticos.

Para modelizar las cinco categorías de estado de salud autopercibida se ha optado por la estimación de un modelo probit ordenado. Este modelo se expresa en términos de una variable latente no observable $\left(h_{i}^{*}\right)$ que es interpretada como "estado real de salud" y es observada a través de la variable que recoge las categorías de estado de salud del individuo $\left(h_{i}\right)$. El modelo se especifica de acuerdo con la siguiente expresión:

$$
h_{i}^{*}=\propto+\beta_{1} \text { tresid } 10_{i}+\sum_{k} \gamma_{k} x_{k, i}+\varepsilon_{i}, \text { con } i=1, \ldots, N
$$

donde tresid $10_{i}$ indica que el individuo lleva residiendo en el país menos de diez años, $x_{k, i}$ es un vector de variables socioeconómicas y demográficas, y $\varepsilon_{i}$ el término error. Los valores $\mu_{1}, \mu_{2}, \mu_{3}$ y $\mu_{4}$ representan los puntos de corte entre las diferentes categorías de salud autopercibida por los individuos:

$$
h_{i}=\left\{\begin{array}{c}
1 \text { (muy bueno), si }-\infty \leq h_{i}^{*}<\mu_{1} \\
2 \text { (bueno), si } \mu_{1} \leq h_{i}^{*}<\mu_{2} \\
3 \text { (regular), si } \mu_{2} \leq h_{i}^{*}<\mu_{3} \\
4 \text { (malo), si } \mu_{3} \leq h_{i}^{*}<\mu_{4} \\
5 \text { (muy malo), si } \mu_{4} \leq h_{i}^{*}<\infty
\end{array}\right.
$$

La modelización de los indicadores binarios de estado de salud (limitaciones de actividad y trastornos crónicos) se realiza a partir de modelos probit. La variable $y_{i}^{*}$ no se observa, pero sí $y_{i}$, que indica la presencia de limitaciones o el padecimiento de problemas crónicos. La probabilidad de tener limitaciones o trastornos de tipo crónico se asume como una función de las características socioeconómicas y demográficas $\left(x_{k, i}\right)$ y el tiempo de residencia en España (tresid $\left.10_{i}\right)$. Los modelos se especifican de acuerdo a la siguiente expresión:

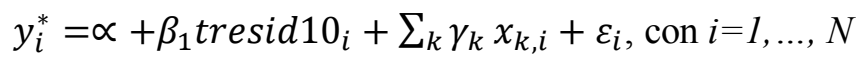

Los modelos probit presentan una variable dependiente que toma el valor 1 cuando el individuo declara padecer limitaciones o trastornos crónicos.

$$
y_{i}=\left\{\begin{array}{l}
1 \text { si } y_{i}^{*}>0 \\
0, \text { otro caso }
\end{array}\right.
$$

\section{Población de estudio}

El análisis empírico se realiza utilizando datos de la Encuesta Nacional de Salud 2011-2012 (julio 2011-junio 2012) realizada por el Ministerio de Sanidad, Servicios Sociales e Igualdad, en colaboración con el Instituto Nacional de 
Estadística (INE, 2013). Dicha encuesta recoge información sanitaria referida a 21.508 hogares con población residente en todo el territorio español. La encuesta tiene un tamaño muestral de 26.502 entrevistas, de las que 21.007 corresponden a personas adultas (15 o más años) y el resto a menores, a través de sus progenitores o tutores. En nuestro caso se ha utilizado únicamente la muestra de adultos.

La ENSE incluye información relevante que abarca aspectos sociodemográficos, de estado de salud, utilización de los servicios sanitarios y determinantes de la salud. La encuesta permite identificar si el adulto entrevistado ha nacido en España o en el extranjero, así como su nacionalidad. Asimismo, esta última edición incluye una variable especialmente relevante para el estudio del estado de salud del inmigrante, su trayectoria y la utilización de servicios sanitarios, como es el número de años que llevan residiendo en nuestro país. Esta información, no recogida en ediciones anteriores de la encuesta, nos permitirá conocer, tanto las diferencias en términos de salud entre ambas poblaciones, como si éstas tenderán a converger o divergir temporalmente.

Excluyendo observaciones con valores perdidos en variables de interés, el colectivo de población inmigrante estaría compuesto por 1.717 individuos que declaran haber nacido en el extranjero. Sin embargo, hemos excluido del mismo a 239 individuos que llegaron a nuestro país en edades tempranas. En este caso, podemos esperar que esta población tenga experiencias similares a la población nacional, en cuanto a hábitos culturales, sociales y ambientales, así como en términos de acceso a los servicios de salud (Nolan, 2012). Nuestro estudio contaría, por tanto, con 1.488 individuos que han nacido en el extranjero y han llegado a nuestro país con 15 o más años de edad.

\section{Variables de estudio}

\section{Variables dependientes}

Considerando las distintas dimensiones de la salud, nuestras variables dependientes están formadas por tres indicadores habitualmente utilizados en la literatura y que han demostrado comportarse como buenos predictores de la misma. La primera, es un indicador del estado de salud percibido en los últimos 12 meses por parte del entrevistado. Lejos de ser un indicador objetivo de salud, el estado de salud percibido refleja las condiciones, experiencias y percepciones particulares del individuo, mostrando una fuerte relación con la morbilidad y la mortalidad (Schultz, 2005). No obstante, es preciso puntualizar que, aun considerándose un buen indicador de morbilidad y mortalidad, diferente literatura argumenta que la percepción del estado de salud varía en función de la edad, de la situación socioeconómica y de otra serie de características (McDonald y Kennedy, 2004). La respuesta a dicha pregunta se categoriza en la encuesta en 
5 opciones, correspondiendo 1 a "muy bueno" y 5 a "muy mal" estado de salud percibido.

En segundo lugar, consideramos una variable dicotómica relacionada con el padecimiento de enfermedad o problema de salud crónico o de larga duración. En tercer lugar, nos referimos a si el individuo encuestado presenta limitaciones en las actividades de la vida diaria por problemas de salud en los últimos 6 meses. En este caso, hemos construido una variable dicotómica si la respuesta es afirmativa (gravemente limitado y limitado pero no gravemente), frente a nada limitado. Estos dos últimos indicadores, sobre todo el indicador relacionado con la existencia de limitaciones, al no estar basados en percepciones individuales o factores socioeconómicos, se puede decir que son indicadores que tienen una menor probabilidad de contener sesgos (Strauss et al., 1995).

\section{Tabla 1}

Características referidas al estado de salud de las poblaciones nativa e inmigrante (\%) Valores ajustados por edad ${ }^{a}$

\begin{tabular}{|c|c|c|c|c|c|c|c|c|c|c|c|c|c|c|}
\hline & \multicolumn{10}{|c|}{ Autovaloración Estado de Salud } & \multirow{2}{*}{\multicolumn{2}{|c|}{$\begin{array}{l}\text { Enfermedad } \\
\text { crónica }\end{array}$}} & \multirow{2}{*}{\multicolumn{2}{|c|}{$\begin{array}{l}\text { Limitación } \\
\text { actividades } \\
\text { diarias }\end{array}$}} \\
\hline & \multicolumn{2}{|c|}{ Muy bueno } & \multicolumn{2}{|c|}{ Bueno } & \multicolumn{2}{|c|}{ Regular } & \multicolumn{2}{|c|}{ Malo } & \multicolumn{2}{|c|}{ Muy Malo } & & & & \\
\hline & $\mathbf{H}$ & $M^{\mathrm{b}}$ & $\mathrm{H}$ & $\mathbf{M}$ & $\mathbf{H}$ & $M$ & $\mathbf{H}$ & M & $\mathbf{H}$ & $M$ & $\mathbf{H}$ & $\mathbf{M}$ & $\mathbf{H}$ & M \\
\hline Nacional & 15,14 & 11,78 & 52,50 & 42,52 & 23,02 & 30,56 & 7,70 & 11,82 & 1,63 & 3,31 & 48,50 & 61,03 & 20,95 & 33,23 \\
\hline Inmigrantes & 26,31 & 19,72 & 52,99 & 51,63 & 17,29 & 22,15 & 2,83 & 5,63 & 0,59 & 0,87 & 24,84 & 39,66 & 11,60 & 18,90 \\
\hline
\end{tabular}

a Las tasas ajustadas por edad, utilizan la estructura de edad de la muestra completa, considerada como la distribución estándar de edades.

${ }^{b} \mathrm{H}=$ hombre, $\mathrm{M}=$ mujer .

Fuente: Elaboración propia a partir de datos de la ENSE 2011-2012 (INE, 2013).

\section{Tabla 2}

Características referidas al estado de salud de la población inmigrante en función del número de años desde la migración (\%) Valores ajustados por edad ${ }^{a}$

\begin{tabular}{|c|c|c|c|c|c|c|c|c|c|c|c|c|c|c|}
\hline & \multicolumn{10}{|c|}{ Autovaloración Estado de Salud } & \multirow{2}{*}{\multicolumn{2}{|c|}{$\begin{array}{l}\text { Enfermedad } \\
\text { crónica }\end{array}$}} & \multirow{2}{*}{\multicolumn{2}{|c|}{$\begin{array}{c}\text { Limitación } \\
\text { actividades } \\
\text { diarias }\end{array}$}} \\
\hline & \multicolumn{2}{|c|}{ Muy bueno } & \multicolumn{2}{|c|}{ Bueno } & \multicolumn{2}{|c|}{ Regular } & \multicolumn{2}{|c|}{ Malo } & \multicolumn{2}{|c|}{ Muy Malo } & & & & \\
\hline & $\mathbf{H}$ & $M^{b}$ & $\mathbf{H}$ & M & $\mathbf{H}$ & M & $\mathbf{H}$ & M & $\mathbf{H}$ & M & $\mathbf{H}$ & M & \begin{tabular}{|l|}
$\mathbf{H}$ \\
\end{tabular} & $\mathbf{M}$ \\
\hline Menos 10 años & 30,32 & 21,25 & 55,88 & 55,29 & 11,29 & 19,83 & 2,51 & 2,38 & - & 1,25 & 17,23 & 31,40 & 8,96 & 14,50 \\
\hline 10 años y más & 15,40 & 11,98 & 52,43 & 42,69 & 23,01 & 30,88 & 7,54 & 11,73 & 1,61 & 3,23 & 50,64 & 60,64 & 20,71 & 32,94 \\
\hline
\end{tabular}

a Las tasas ajustadas por edad, utilizan la estructura de edad de la muestra completa, considerada como la distribución estándar de edades.

${ }^{b} \mathrm{H}=$ hombre, $\mathrm{M}=$ mujer .

Fuente: Elaboración propia a partir de datos de la ENSE 2011-2012 (INE, 2013).

Como puede verse en la Tabla 1, los inmigrantes reportan mejores niveles de salud que la población nacional en los tres indicadores considerados, aprecián- 
dose mayores diferencias en las variables referidas al padecimiento de enfermedades crónicas o de larga duración, y limitaciones en las actividades de la vida diaria. El estado de salud también difiere en función del número de años desde la migración. Aquellos inmigrantes que llevan 10 o más años en nuestro país reportan estados de salud similares a la población nacional, mientras que los inmigrantes más recientes presentan mejores resultados en los indicadores (Tabla 2). Las diferencias se mantienen por sexos, reportando las mujeres peor estado de salud que los hombres.

\section{Variables independientes}

En cuanto a las variables independientes, la característica de ser inmigrante se registra en la encuesta en base a su país de nacimiento. Así, consideramos inmigrantes a los que han nacido fuera de España, asignándoles el valor 1 en dicha variable. Con el objetivo de analizar el proceso de asimilación del colectivo de inmigrantes respecto a la población nacional, en cuanto a estados salud, se ha considerado el tiempo de residencia en España declarado por el propio individuo en la encuesta a través de la pregunta ¿Cuántos años lleva residiendo en España? Se ha definido la variable dicotómica nacido fuera de España y que reside en España hace menos de 10 años, y la variable continua de tiempo de residencia en España (García, 2007; Nolan, 2012; Wu u Schimmele, 2005). La segunda variable se ha utilizado en una submuestra de población inmigrante, dada la relevancia de conocer el impacto del tiempo en los estados de salud declarados junto con su evolución temporal.

El lugar de nacimiento es un factor determinante del estado de salud, al recoger diferencias no observables en los cuidados prenatales, neonatales y de infancia; factores ambientales, y diferencias en actitudes sociales y culturales sobre comportamientos relacionados con la salud y la utilización de servicios sanitarios (McDonald y Kennedy, 2004). En nuestras estimaciones también se han tenido en cuenta 5 categorías que representan el país de nacimiento de los individuos catalogados como inmigrantes, agrupados por regiones.

Asimismo, la población inmigrante presenta diferencias respecto a otras características, como pueden ser la edad, el sexo, el nivel de educación, su actividad principal, etc. Los inmigrantes, en media, son más jóvenes y presentan mejores niveles de educación que la población nativa, aunque realizan trabajos con menor nivel de cualificación. En ausencia de un indicador de renta a nivel individual, se ha optado por utilizar, como una aproximación al mismo, una variable que indica si el individuo tiene en vigor un contrato laboral, así como una variable representativa del tipo de trabajo realizado y el nivel de cualificación requerido para el mismo. La Tabla 3 presenta las estadísticas descriptivas de las variables independientes utilizadas en las regresiones desarrolladas en este estudio. 


\section{Tabla 3}

Definición de las variables independientes y estadísticas descriptivas (\%)

\begin{tabular}{|c|c|c|c|}
\hline Variable & Definición & Nativo & Inmigrante \\
\hline Inmigrante & 1, si nació en el extranjero, 0 si nació en España & - & 100,00 \\
\hline Años migración & Número de años residiendo en España & - & 10,72 \\
\hline Residencia $<10$ años & 1, si lleva menos de 10 años residiendo en España & - & 54,21 \\
\hline Edad1 & 1 , si tiene entre 15 y 24 años, 0 en otro caso & 7,49 & 6,26 \\
\hline Edad2 & 1 , si tiene entre 25 y 34 años, 0 en otro caso & 11,98 & 27,78 \\
\hline Edad3 & 1 , si tiene entre 35 y 44 años, 0 en otro caso & 17,88 & 30,2 \\
\hline Edad4 & 1 , si tiene entre 45 y 54 años, 0 en otro caso & 16,87 & 19,35 \\
\hline Edad5 & 1, si tiene entre 55 y 64 años, 0 en otro caso & 15,71 & 9,77 \\
\hline Edad6 & 1 , si tiene 65 o más años, 0 en otro caso & 30,06 & 6,64 \\
\hline Hombre & 1 , si tiene sexo masculino, 0 en otro caso & 45,94 & 46,10 \\
\hline Casado & 1 , si está casado, 0 en otro caso & 52,44 & 54,19 \\
\hline Separado & 1, si está separado, 0 en otro caso & 6,3 & 8,38 \\
\hline Viudo & 1, si está viudo, 0 en otro caso & 13,96 & 3,71 \\
\hline Edu1 & 1, si no ha completado la educación primaria, 0 en otro caso & 15,34 & 6,90 \\
\hline Edu2 & 1, si ha completado la educación primaria, 0 en otro caso & 13,36 & 5,81 \\
\hline Edu3 & $\begin{array}{l}\text { 1, si ha completado la } 1^{\text {a }} \text { etapa de secundaria o FP de grado } \\
\text { medio, } 0 \text { en otro caso }\end{array}$ & 40,36 & 36,59 \\
\hline Edu4 & $\begin{array}{l}\text { 1, si ha completado el bachillerato o FP de grado superior, } 0 \text { en } \\
\text { otro caso }\end{array}$ & 30,94 & 50,7 \\
\hline Edu5 & 1, si tiene estudios universitarios, 0 en otro caso & 14,87 & 16,92 \\
\hline Trabaja & 1 , si tiene algún contrato laboral, 0 en otro caso & 40,55 & 53,7 \\
\hline Clase1 & 1 , si es un trabajador no cualificado, 0 en otro caso & 13,87 & 28,68 \\
\hline Municipio & $\begin{array}{l}\text { 1, si reside en un municipio de menos de } 10.000 \text { habitantes, } 0 \\
\text { en otro caso }\end{array}$ & 24,63 & 15,84 \\
\hline Latinoamérica & $\begin{array}{l}\text { 1, si su país de nacimiento se sitúa en Latinoamérica, } 0 \text { en otro } \\
\text { caso }\end{array}$ & - & 45,40 \\
\hline Unión Europea & 1, si su país de nacimiento pertenece a la UE- 27,0 en otro caso & - & 29,31 \\
\hline África & 1, si su país de nacimiento se sitúa en África, 0 en otro caso & - & 16,28 \\
\hline Europa & $\begin{array}{l}\text { 1, si su país de nacimiento pertenece a un país europeo que no } \\
\text { pertenezca a la } U E, 0 \text { en otro caso }\end{array}$ & - & 3,45 \\
\hline Asia & 1, si su país de nacimiento se sitúa en Asia, 0 en otro caso & - & 4,73 \\
\hline
\end{tabular}

Fuente: Elaboración propia a partir de datos de la ENSE 2011-2012 (INE, 2013).

\section{RESULTADOS}

En la Tabla 4 se presentan los coeficientes estimados para los tres indicadores de salud considerados: salud autopercibida (modelo 1), padecimiento de problemas de salud crónicos o de larga duración (modelo 2) y limitaciones para las actividades de la vida diaria (modelo 3). Con la finalidad de interpretar de forma cuantitativa los resultados anteriores, en la Tabla 5 se recogen los efectos marginales para las variables de interés. 


\section{Tabla 4}

Especificaciones básicas de estado de salud en relación a la condición de inmigrante y el tiempo de residencia en España

\begin{tabular}{|c|c|c|c|c|c|c|}
\hline & (1) & (2) & (3) & (1) & (2) & (3) \\
\hline Edad2 (25-34 años) & $\begin{array}{c}0,433^{\star \star \star} \\
(0,038)\end{array}$ & $\begin{array}{c}0,354^{\star \star \star} \\
(0,049)\end{array}$ & $\begin{array}{c}0,301^{* * *} \\
(0,06)\end{array}$ & $\begin{array}{c}0,432^{* * *} \\
(0,038)\end{array}$ & $\begin{array}{c}0,357^{\star * *} \\
(0,048)\end{array}$ & $\begin{array}{c}0,314^{\star * *} \\
(0,059)\end{array}$ \\
\hline Edad3 (35-44 años) & $\begin{array}{c}0,689^{* * *} \\
(0,037)\end{array}$ & $\begin{array}{c}0,611^{* * *} \\
(0,049)\end{array}$ & $\begin{array}{c}0,491^{* * *} \\
(0,059)\end{array}$ & $\begin{array}{c}0,685^{\star * *} \\
(0,037)\end{array}$ & $\begin{array}{c}0,600^{* * *} \\
(0,046)\end{array}$ & $\begin{array}{c}0,509^{\star * *} \\
(0,056)\end{array}$ \\
\hline Edad4 (45-54 años) & $\begin{array}{c}0,922^{\star * *} \\
(0,037)\end{array}$ & $\begin{array}{c}0,943^{\star * *} \\
(0,049)\end{array}$ & $\begin{array}{c}0,682^{* * *} \\
(0,059)\end{array}$ & $\begin{array}{c}0,918^{* * *} \\
(0,037)\end{array}$ & $\begin{array}{c}0,932^{* * *} \\
(0,046)\end{array}$ & $\begin{array}{c}0,711^{* * *} \\
(0,055)\end{array}$ \\
\hline Edad5 (55-64 años) & $\begin{array}{c}1,090^{* * *} \\
(0,037)\end{array}$ & $\begin{array}{c}1,201^{* * *} \\
(0,05)\end{array}$ & $\begin{array}{c}0,746^{* * *} \\
(0,059)\end{array}$ & $\begin{array}{c}1,086^{* * *} \\
(0,037)\end{array}$ & $\begin{array}{c}1,202^{* * *} \\
(0,046)\end{array}$ & $\begin{array}{c}0,774^{* * *} \\
(0,054)\end{array}$ \\
\hline Edad6 (65 o más años) & $\begin{array}{c}1,220^{\star \star \star} \\
(0,035)\end{array}$ & $\begin{array}{c}1,432^{\star * *} \\
(0,05)\end{array}$ & $\begin{array}{c}0,936^{* * *} \\
(0,059)\end{array}$ & $\begin{array}{c}1,217^{* \star *} \\
(0,035)\end{array}$ & $\begin{array}{c}1,431^{* * *} \\
(0,044)\end{array}$ & $\begin{array}{c}0,966^{* * *} \\
(0,552)\end{array}$ \\
\hline Hombre & $\begin{array}{c}-0,158^{* * *} \\
(0,015)\end{array}$ & $\begin{array}{c}-0,186^{\star * *} \\
(0,019)\end{array}$ & $\begin{array}{c}-0,225^{\star \star *} \\
(0,021)\end{array}$ & $\begin{array}{c}-0,158^{\star * *} \\
(0,155)\end{array}$ & $\begin{array}{c}-0,181^{* * *} \\
(0,018)\end{array}$ & $\begin{array}{c}-0,228^{* * *} \\
(0,020)\end{array}$ \\
\hline Trabaja & $\begin{array}{c}-0,258^{\star * *} \\
(0,019)\end{array}$ & $\begin{array}{c}-0,245^{\star \star *} \\
(0,023)\end{array}$ & $\begin{array}{c}-0,272^{\star * *} \\
(0,027)\end{array}$ & $\begin{array}{c}-0,258^{* * *} \\
(0,019)\end{array}$ & $\begin{array}{c}-0,242^{\star * *} \\
(0,023)\end{array}$ & $\begin{array}{c}-0,270^{\star * *} \\
(0,027)\end{array}$ \\
\hline Edu2 (Educación primaria) & $\begin{array}{c}-0,192^{\star * *} \\
(0,028)\end{array}$ & $\begin{array}{c}-0,034 \\
(0,037)\end{array}$ & $\begin{array}{c}-0,106^{\star * *} \\
(0,036)\end{array}$ & $\begin{array}{c}-0,192^{\star * \star} \\
(0,028)\end{array}$ & $\begin{array}{l}-0,041 \\
(0,036)\end{array}$ & $\begin{array}{c}-0,161^{* * *} \\
(0,035)\end{array}$ \\
\hline $\begin{array}{r}\text { Edu3 (1 }{ }^{a} \text { etapa de secundaria } \\
\text { o FP de grado medio) }\end{array}$ & $\begin{array}{c}-0,361^{* * *} \\
(0,025)\end{array}$ & $\begin{array}{l}-0,079^{* *} \\
(0,033)\end{array}$ & $\begin{array}{c}-0,271^{* * *} \\
(0,033)\end{array}$ & $\begin{array}{c}-0,362^{* * *} \\
(0,025)\end{array}$ & $\begin{array}{c}-0,091^{* *} \\
(0,031)\end{array}$ & $\begin{array}{c}-0,289^{* * *} \\
(0,031)\end{array}$ \\
\hline $\begin{array}{r}\text { Edu4 (Bachiller o FP grado } \\
\text { Superior) }\end{array}$ & $\begin{array}{c}-0,538^{* * *} \\
(0,030)\end{array}$ & $\begin{array}{l}-0,152^{* * *} \\
(0,038)\end{array}$ & $\begin{array}{c}-0,379^{* * *} \\
(0,041)\end{array}$ & $\begin{array}{l}-0,539^{* *} \\
(0,030)\end{array}$ & $\begin{array}{c}-0,178^{* * *} \\
(0,037)\end{array}$ & $\begin{array}{c}-0,405^{\star * *} \\
(0,039)\end{array}$ \\
\hline Edu5 (Estudios universitarios) & $\begin{array}{c}-0,135^{\star * *} \\
(0,027)\end{array}$ & $\begin{array}{l}-0,022 \\
(0,033)\end{array}$ & $\begin{array}{r}-0,051 \\
(0,04)\end{array}$ & $\begin{array}{c}-0,134^{* * *} \\
(0,027)\end{array}$ & $\begin{array}{c}0,026 \\
(0,032)\end{array}$ & $\begin{array}{l}-0,056 \\
(0,039)\end{array}$ \\
\hline Inmigrante & $\begin{array}{l}-0,049^{*} \\
(0,029)\end{array}$ & $\begin{array}{l}-0,262^{\star * *} \\
(0,037)\end{array}$ & $\begin{array}{l}-0,102^{\star *} \\
(0,044)\end{array}$ & 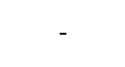 & 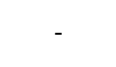 & 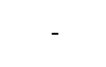 \\
\hline Residencia < 10 años & - & - & - & $\begin{array}{l}-0,079^{*} \\
(0,039)\end{array}$ & $\begin{array}{c}-0,351^{* * *} \\
(0,051)\end{array}$ & $\begin{array}{l}-0,016^{* *} \\
(0,060)\end{array}$ \\
\hline $\mathbf{N}$ & 20.690 & 20.674 & 20.683 & 20.690 & 20.674 & 20.683 \\
\hline Log-L & $-23703,41$ & -12385.78 & $-9944,48$ & $-23702,78$ & $-12385,7$ & $-9944,4$ \\
\hline Pseudo $\mathbf{R}^{2}$ & 0,091 & 0,134 & 0,099 & 0,091 & 0,134 & 0,099 \\
\hline
\end{tabular}

*** Significativa al 1\%; ** Significativa al 5\%; * Significativa al $10 \%$,

${ }^{a}$ Error estándar entre paréntesis

${ }^{b}$ Las variables dependientes de los modelos (1), (2) y (3) son, respectivamente, autovaloración del estado de salud (5 categorías), padecimiento de problema de salud crónico y presentar limitaciones en las actividades de la vida diaria por problemas de salud.

${ }^{c}$ Las categorías de referencia son edad 15-24 años, ser mujer, no tener contrato de trabajo en vigor y nivel de educación primaria incompleta. Asimismo, se han considerado como variables de control el país de nacimiento de la población inmigrante, el estado civil, el tamaño del municipio de residencia y la cualificación del trabajo desempeñado.

Fuente: Elaboración propia.

Respecto al estado de salud declarado por el individuo, ser inmigrante deriva, de forma significativa, en una mayor probabilidad de declarar un mejor estado de salud. La probabilidad de declarar un estado de salud "muy bueno" o "bueno" en el caso de la población inmigrante, es de un $1,1 \%$ y $0,5 \%$ superior a la de la población nacional, mientras que las probabilidades de percibir un estado de salud regular, malo o muy malo disminuyen en 1,$0 ; 0,4$ y $0,1 \%$, respectivamente. Por lo que respecta a los indicadores objetivos de estado de salud, 
la condición de ser inmigrante repercute en una menor probabilidad de declarar problemas de salud crónicos $(10,23 \%)$ y limitaciones para las actividades de la vida diaria $(2,75 \%)$.

Asimismo, según podemos apreciar en los resultados obtenidos, la probabilidad de declarar peor estado de salud aumenta en todos los indicadores considerados con el tiempo de residencia en España. En el caso del indicador de salud autopercibida, la probabilidad de declarar un estado de salud "muy buena" o "buena" aumenta en un 1,8\% y $0,8 \%$, respectivamente, para la población que reside en nuestro país desde hace menos de 10 años. Para estados de salud regulares, malos o muy malos la probabilidad desciende en un $1,7 \%, 0,7 \%$ y $0,2 \%$, respectivamente para este mismo colectivo. La probabilidad de padecer problemas de salud crónicos disminuye para los inmigrantes que llevan menos de 10 años en nuestro país en un 13,5\%, y en un $4,4 \%$ en el caso de limitaciones de la vida diaria. En consecuencia, los inmigrantes tienden a declarar un peor estado de salud, y presentan una mayor incidencia de problemas de crónicos y limitaciones, a medida que aumenta su tiempo de estancia en nuestro país, resultados consistentes con la existencia del HIE.

\section{Tabla 5}

Efectos marginales en las variables de interés

\begin{tabular}{lcccccccc}
\hline \multicolumn{7}{c}{ Salud autopercibida (\%) } \\
\hline & Muy bueno & Bueno & Regular & Malo & Muy malo & $\begin{array}{c}\text { Enfermedades } \\
\text { crónicas (\%) }\end{array}$ & $\begin{array}{c}\text { Limitaciones } \\
\text { (\%) }\end{array}$ \\
\hline Inmigrante & 1,1 & 0,5 & $-1,0$ & $-0,4$ & $-0,1$ & $-10,23$ & $-2,75$ \\
Residencia < 10 años & 1,8 & 0,8 & $-1,7$ & $-0,7$ & $-0,2$ & $-13,5$ & $-4,4$ \\
\hline
\end{tabular}

${ }^{a}$ Efectos marginales calculados en los valores medios de las variables (Greene, 2005). Los efectos marginales de las variables binarias representan el cambio en la probabilidad esperada cuando la variable pasa de 0 a 1.

Fuente: Elaboración propia.

La Tabla 4 también recoge los efectos obtenidos en otras variables explicativas relevantes, presentando los comportamientos esperados. En las tres primeras ecuaciones, encontramos que las variables que representan la edad del individuo son significativas al determinar el estado de salud. Así, se observa un gradiente de edad que refleja que el estado de salud empeora a medida que la edad avanza, según nos muestran los coeficientes de los diferentes intervalos considerados. El género también se muestra como una variable representativa, resultando mejor estado de salud para el sexo masculino que para el femenino. El tener un empleo favorece, en términos de probabilidad, el declarar un mejor estado de salud y disminuye las limitaciones y el padecimiento de enfermedades crónicas. Este resultado estaría en línea con los estudios que analizan el gradiente socioeconómico y el estado de salud, que presentan evidencia respecto a 
que mayores niveles de ingresos están asociados a mejores resultados en niveles de salud (Smith, 2004; Adams et al., 2003). En este sentido, debemos tener en cuenta que el modelo estimado no es un modelo estructural de estado de salud, y por lo tanto, nuestro objetivo no es dar una interpretación causal de los estimadores, puesto que algunos de los mismos podrían estar condicionados, además, por el estado de salud. Es necesario, no obstante, incluir dichas variables, para que el coeficiente objeto de estudio no se vea afectado por la ausencia de variables relevantes.

El nivel educativo también es una variable considerada por la literatura como determinante del estado de salud individual (Grossman, 2000; Smith, 2004). De los resultados obtenidos, la educación parece favorecer mejores estados de salud a medida que se avanza en etapas formativas, con excepción de haber completado estudios universitarios, que no se muestra como representativo en alguno de los indicadores y no sigue la misma evolución que los demás niveles considerados.

Por último, se han estimado los modelos anteriores solo para la submuestra de inmigrantes, incluyendo como variable explicativa las variables tiempo desde su llegada a España y su cuadrado. Los resultados muestran que el tiempo de residencia influye de forma significativa y negativa sobre los estados de salud percibida muy buena y buena, y de forma positiva sobre las restantes categorías. Respecto a los indicadores objetivos de estado de salud, un año adicional de residencia aumenta la probabilidad de padecer enfermedades crónicas y sufrir limitaciones de las actividades diarias, en un 1,12 y en un $0,70 \%$ respectivamente. Los resultados, respecto a la evolución de dicho efecto a tasas decrecientes, sugieren que los estados de salud de los inmigrantes convergen a los de la población autóctona con los años de residencia en España. ${ }^{1}$

\section{CONCLUSIONES}

El objetivo de este estudio ha sido analizar la trayectoria de salud de la población inmigrante en función de los años de estancia en nuestro país, aportando evidencia empírica sobre lo que la literatura conoce como el Healthy Inmigrant Effect. Dicho efecto, supone una convergencia del estado de salud de la población inmigrante hacia el de la población nacional, y esta convergencia en estados de salud tendrá, como factor determinante, el tiempo que el inmigrante lleve residiendo en el país de destino. La literatura existente presenta evidencia respecto a que la salud de los inmigrantes, en general, es mejor que la de la población a su llegada al país de destino $(H S)$, pero ésta se va deteriorando a medida que pasa el tiempo (HIE).

\footnotetext{
${ }^{1}$ Resultados de las estimaciones disponibles bajo petición a los autores.
} 
Para desarrollar dicho análisis se han utilizado datos de la última ENSE, que al incluir la pregunta sobre el tiempo de residencia, a los individuos que han declarado haber nacido fuera de España, permite relacionar dicha variable con su estado de salud. Se han considerado tres indicadores de estado de salud, frecuentemente utilizados por la literatura: estado de salud autopercibido, padecimiento de una enfermedad crónica o de larga duración, y padecimiento de limitaciones en las actividades de la vida diaria. Se han analizado, en primer lugar, las diferencias presentadas para dichos indicadores entre la población nacional y la población inmigrante, después de controlar por un conjunto de variables socieconómicas y demográficas. Posteriormente, se ha comprobado la significación del tiempo de residencia en el país de destino, por parte de la población inmigrante, como variable que puede influir en la dinámica de su estado de salud en el periodo posterior a la migración.

Los resultados obtenidos indican que los inmigrantes reportan un mejor estado de salud que la población nacional, efecto encontrado en los todos los indicadores de salud considerados. Asimismo, obtenemos evidencia a favor del $H I E$, dado que el tiempo de residencia en el país de destino aparece como determinante significativo del estado de salud de la población inmigrante y, por lo tanto, determinante de una evolución hacia un empeoramiento en su estado de salud. Dicho efecto va evolucionando a tasa decreciente, a medida que transcurre el tiempo de estancia, convergiendo con el estado de salud de la población nacional.

Respecto a investigaciones sobre el $H I E$ en países que presentan una trayectoria reciente en recibir inmigrantes, como la desarrollada por Nolan (2012) para Irlanda, nuestros resultados muestran un efecto mayor del periodo de residencia sobre las variables de resultado de salud. En este punto, es preciso considerar que la realidad de la inmigración, en cuanto a su origen, es distinta para ambos países. La población extranjera asentada en Irlanda procede, en su mayoría, de países con características socioculturales similares a Irlanda, mientras que la población inmigrante en España es diversa en cuanto a su origen, y responde a unas realidades sociales, culturales y económicas que, en muchos casos, difieren de las españolas.

Debemos señalar que el análisis de la trayectoria de salud de la población inmigrante en función del tiempo de residencia en el país presenta algunas limitaciones debidas al uso de datos de corte transversal. En este sentido, no podemos considerar determinadas características inobservables que pueden diferir entre inmigrantes llegados en diferentes períodos temporales. Tampoco debemos olvidar los posibles sesgos derivados de la muestra de población inmigrante que permanece en nuestro país, si se trata de los individuos más saludables y con mejores condiciones en el mismo. 
El tipo de inmigración que llega a España, fundamentalmente de tipo económico, también permite considerar la existencia de una selección positiva en términos de salud. Todo esto, unido a la ausencia de información específica sobre estados de salud de los individuos, características socioeconómicas o información sobre acceso a servicios de salud en etapas anteriores a la migración, y justo a su llegada al país de destino, no permite explicar la totalidad de factores determinantes del HIE. En todo caso, la disminución en el nivel de salud de la población inmigrante, y su convergencia hacia los niveles de la población nacional, refleja una combinación de factores, como podría ser el caso de un proceso de aculturación y un mayor diagnóstico de problemas de salud.

Desde una perspectiva de política sanitaria, el abordaje de las diferencias en salud en la población extranjera y su evolución temporal, se configura como una cuestión relevante en orden a intentar satisfacer unas necesidades de atención que se muestran cambiantes con el tiempo, y que dependen de determinados factores y características socioeconómicas de los individuos.

\section{REFERENCIAS BIBLIOGRÁFICAS}

ADAMS, P.; HURD, M. D.; MCFADDEN, D.; MERRILL, A. y RIBEIRO, T. (2003). "Healthy, wealthy and wise? Tests for direct causal paths between health and socioeconomic status" en Journal of Econometrics, 112, pp. 3-56.

ANTECOL, H. y BEDARD, K. (2005). "Unhealthy Assimilation: Why Do Immigrants Converge to American Health Status Levels?" en IZA Discussion Paper, 1654. Bonn: IZA.

BIDDLE, N.; KENNEDY, S. y MCDONALD, J.T. (2007). "Health Assimilation Patterns Amongst Australian Immigrants" en Economic Record, 83(260), pp. 16-30.

CANTARERO, D. y PASCUAL, M. (2008). "Salud y utilización en la población inmigrante: una revisión del caso español". En Atención Sanitaria y Nuevas Necesidades Poblacionales: Inmigración y Demanda. Santiago de Compostela: FEGAS.

CARRASCO, G.P.; GIL DE MIGUEL, A.; HERNÁNDEZ, B.V. y JIMÉNEZ, G.R. (2007). "Health profiles, lifestyles and use of health resources by the immigrant population resident in Spain" en European Journal of Public Health, 17(5), pp. 503-507.

CHEN, J.; EDWARD, N.G. y RUSSELL, W. (1996). "The Health of Canada's Immigrants in 994-95." en Health Reports, 7(4), pp. 33-45. 
DUNN, J. y DYCK, I. (2000). "Social determinants of health in Canada's immigrant population: results from the national population health survey" en Social Science and Medicine, 51, pp. 1573-1593.

FRISBIE, W.P.; CHO, Y. y HUMMER, R.A. (2001). "Immigration and the health of Asian and Pacific Islanderadults in the United States" en American Journal of Epidemiology, 153(4), pp. 372-80.

GARCÍA-GÓMEZ, P. (2007). "Salud y utilización de los recursos sanitarios: Un análisis de las diferencias y similitudes entre población inmigrante y autóctona" en Presupuesto y Gasto Público, 49(4), pp. 67-85.

GREENE, W.H. (2005). Econometric Analysis ( $5^{\text {th }}$ Ed.). New York: PrenticeHall.

GOEL, M.S.; MCCARTHY, E.P.; PHILLIPS, R.S. y WEE, C.C. (2004). "Obesity among US immigrant subgroups by duration of residence" en Journal of the American Medical Association, 292(23), pp. 2860-2867.

GÓMEZ, S.L.; KELSEY, J.L.; GLASER, S.L.; LEE, M.M. y SIDNEY, S. (2004). "Immigration and acculturation in relation to health and health-related risk factors among specific Asian subgroups in a Health Maintenance Organisation" en American Journal of Public Health, 94(11), pp. 1977-1984.

GROSSMAN, M. (2000). "The human capital model". En A. J. Culyer y J. P. Newhouse (Eds.): Handbook of health economics (pp. 347-408). Amsterdam: Elsevier.

HAMILTON, T.G. y HUMMER, R.A. (2011). "Immigration and the health of U.S. black adults: does country of origin matter?" en Social Science and Medicine, 73(10), pp. 1551-60

HERNÁNDEZ-QUEVEDO, C. y JIMÉNEZ-RUBIO, D. (2009). "A comparison of the health status and health care utilisation patterns between foreigners and the national population in Spain: new evidence from the Spanish national health survey" en Social Science and Medicine, 69, pp. 370-378.

HOUSE, J.S.; KESSLER, R.C.; HERZOG, A.R.; MERO, R.P.; KINNEY, A.M. y BRESLOW, M.J. (1990). "Age, socioeconomic status and health" en The Milbank Quarterly, 68, pp. 383-411.

HYMAN, I. (2001). "Immigration and health" en Health Policy Working Paper, 01-05. Ottawa: Health Canada.

INSTITUTO NACIONAL DE ESTADÍSTICA (2013). Encuesta Nacional de Salud 2011-2012. Madrid: INE.

INSTITUTO NACIONAL DE ESTADÍSTICA (VARIOS AÑOS). Padrón Municipal: Explotación Estadística. INE. http://www.ine.es/jaxi/menu.do?type= pcaxis\&path $=\% 2 \mathrm{Ft} 20 \% 2 \mathrm{Fe} 245 \&$ file=inebase $\& \mathrm{~L}=0$ [Último acceso: mayo de 2013].

JASSO, G.; MASSEY, D.; ROSENZWEIG, M. y SMITH, J. (2004). "Immigrant Health: Selectivity and Acculturation" en IFS Working Paper, 04/23. London: Institute for Fiscal Studies. 
KENNEDY, S.; MCDONALD, J. y BIDDLE, N. (2006). The healthy immigrant effect and immigrant selection: evidence from four countries. Hamilton: McMaster University.

LAROCHE, M. (2000). "Health status and health services utilisation of Canada's immigrant and non-immigrant populations" en Canadian Public Policy, 26(1), pp. 51-75.

LECLERE, F.B.; LEIF, J. y BIDDLECOM, A.E. (1994). "Health Care Utilization, Family Context, and Adaptation Among Immigrants to the United States" en Journal of Health and Social Behavior, 35(4), pp. 370-384.

MCDONALD, J.T. y KENNEDY, S. (2004). "Insights into the 'healthy immigrant effect': health status and health service use of immigrants to Canada" en Social Science and Medicine, 59, pp. 1613-1627.

MARMOT, M.G.; ADELSTEIN, A.M. y BULUSU, L. (1984). "Lessons from the study of immigrant mortality" en Lancet, 1(8392), pp. 1455-1457.

MARMOT, M.G. y SYME, S.L. (1976). "Acculturation and Coronary Heart Disease in Japanese- Americans" en American Journal of Epidemiology, 104(3), pp. 225-247.

NEWBOLD, B. y DANFORTH, J. (2003). "Health status and Canada's immigrant population" en Social Science and Medicine, 57, pp. 1981-1995.

NOLAN, A. (2012). "The 'healthy immigrant' effect: initial evidence for Ireland" en Health Economics, Policy and Law, 7, pp. 343-362.

PALLONI, A. y ARIAS, E. (2003). "A Re-Examination of the Hispanic Mortality Paradox" en Center for Demography and Ecology Working Paper, 2003-01. University of Wisconsin-Madison.

PALLONI, A. y MORENOFF, J. (2001). "Interpreting the Paradoxical in the 'Hispanic Paradox': Demographic and Epidemiological Approaches". En M. Weinstein, A. Hermalin, and M. Stoto (Eds.): Population Health and Aging (pp. 140-174). New York: New York Academy of Sciences.

PEREZ, C. (2002). "Health Status and Health Behaviour among Immigrants" en Health Reports, 13. Ottawa: Statistics Canada.

RIVERA, B.; CASAL, B.; CANTARERO, D. y PASCUAL, M. (2008). "Adaptación de los servicios de salud a las características específicas y de utilización de los nuevos españoles" en Gaceta Sanitaria, 22(S1), pp. 86-95.

SCHULTZ, T.P. (2005). "Productive benefits of health: evidence from low-income countries". En G. López-Casasnovas, B. Rivera y L. Currais (Eds.): Heath and Economic Growth. Findings and Policy Implications (pp. 257285). Cambridge, MA: The MIT Press.

SMITH, J. P. (2004). "Unravelling the SES health connection" en The Institute for Fiscal Studies Working Paper, 04-02. Londres: The Institute for Fiscal Studies.

SOLSONA, M. y VICIANA, F. (2004). "Claves de la Evolución Demográfica en el Cambio de Milenio" en Gaceta Sanitaria, 18, pp. 8-15. 
SORLIE, P.D.; BACKLUND, E. y KELLER, J. (1995). "U.S. Mortality by Economic, Demographic, and Social Characteristics: The National Longitudinal Mortality Study" en American Journal of Public Health, 85, pp.949-956.

STEPHEN, E.H.; FOOTE, K.; HENDERSHOT, G.E. y SCHOENBORN, C.A. (1994). "Health of the foreign-born population" en Advance Data from Vital and Health Statistics, 241, pp. 1-10.

STRAUSS, J.; GERTLER, P.J.; RAHMAN, O. y FOX, K. (1995). "Gender and life-cycle differentials in the patterns and determinants of adult health". En T.P. Schultz (Ed.): Investment in Women's Human Capital. Chicago: University of Chicago Press.

WISTER, A.V. (2005). Baby Boomer health Dynamics: How are we aging? Toronto: University of Toronto Press.

WU, Z., SCHIMMELE, CH. (2005). "The Healthy Migrant Effect on Depressión: Variation over Time?" en Canadian Studies in Population, 32(2), pp. 271295. 
